\title{
Analysis of Lethal Course of Stroke-Prone SHR
}

\author{
Ichiro Akiguchi, ${ }^{*}$ Ryoichi Horie, ${ }^{\circ * *}$ Yasuo Nara, ${ }^{\circ * * *}$ \\ Yukio YAMORI, ${ }^{\circ * * *}$ and Chuichi KaWAI*
}

In order to clarify mechanisms for the lethal course of stroke in stroke-prone SHR (SHRSP), we employed several patho-physiological and patho-anatomical approaches. We compared these findings with those in humans, and we studied the qualification of SHRSP as a clinical model for stroke in man.

Materials and Methods:

1. Adult male and female SHRSP exhibiting remarkable general and focal signs of stroke such as head enlargement, exophthalmus, general weakness, and quadriparesis were sacrificed to closely observe the topographical relation between the brain and the cranium or dura mater. After fixation with $10 \%$ formalin, slices of the brain were roughly examined, then pieces from the lesions and lower brain stem were embedded in paraffin, sectioned and stained in hematoxylin and cosin or else luxol fast blue and cresyl violet for histological examination.

2. Some of these rats, at the terminal stage of cerebrovascular diseases, were immediately frozen in liquid nitrogen without any pretreatment, and were then decapitated in the frozen state at the level of the lower cervical portion. These heads, together with skull and scalp, were cut vertically in the midline and sectioned serially on a cryostat.

Next the correlation between the brain and the cranium or dura mater was observed macroscopically and photographed.

3. Thermographic respiratory patterns were recorded without anesthesia and restraint through a thermister implanted into the nasal cavity of the animals exhibiting clinical signs of severe stroke.

4. For chronological analysis of the lethal course, blood pressure, pulse rate, EKG, and ECG were simultaneously recorded on a polygraph.

\section{Results with Discussion:}

In these patho-anatomical studies, the most important findings observed in SHRSP with severe stroke lesions were the presence of cerebral herniation (occipital lobe herniation) and cerebellar herniation (uvular herniation) with lower brain stem damage. Then, the brain shift caused by severe cerebrovascular lesions in SHRSP leads to impaction mainly in 2 regions, the incisura tentorii and the foramen magnum. Backward and medialward brain shift at the incisura tentorii induces central herniation and cerebral herniation, which result in midbrain distortion and infarction at the territory of the posterior cerebral artery.

Backward displacement of the brain stem leads to the impaction of the cerebellum and uvula in the foramen magnum, so that the displaced cerebellum compresses the medulla oblongata. As a result of this mechanical compression and vascular insufficiency, secondary lower brain stem lesions develop. By histological

From the Japan Stroke Prevention Center (JSPG), Izumo; Departments of Internal Medicine,* Neurosurgery, ${ }^{* *}$ and Pathology, ${ }^{* *}$ Faculty of Medicine, Kyoto University, Kyoto. 
examination, existence of secondary brain stem hemorrhage and infarction in the dorsomesial part of the lower brain stem were proved. To confirm these pathoanatomical findings, electrophysiological studies for respiratory patterns at the terminal stage were helpful. Thus, the lethal course of stroke in SHRSP seems to be accelerated by at least 2 "vicious circles": 1) Pretentorial mass effect due to severe cerebrovascular lesions induces central herniation (diencephalic shift) and cerebral herniation (occipital lobe herniation). Such herniation compresses the posterior cerebral artery against the tentorium, causing infarction of the occipital lobe and further intensifying the pretentorial mass effect. 2) This pretentorial mass effect induces cerebellar and brainstem deformity and cerebellar herniation, stretching or compressing the vertebrobasilar arteries and their branches. It results in secondary posttentorial lesions. Thus, obstruction of foramen magnum and the resulting disturbances of the cerebrospinal fluid flow all intensify the pretentorial mass effect again. They further accelerate posttentorial lesions, finally causing brain death from severe lower brain stem dysfunction leading to either respiratory arrest or catastrophes of cardiovascular regulation.

\section{Summary:}

In SHRSP at the terminal stage, brain shift and herniation were confirmed to develop further cerebral damage. Cerebellar herniation is the major cause of brainstem dysfunction which results in brain death. These are also the cause of acceleration of brain swelling and the establishment of massive infarction. Generally, the lethal course of stroke in SHRSP corresponds well to that of patients with severe cerebrovascular lesions.

This study was supported by the Science and Technology Agency of Japanese Government, Ministry of Education, National Institutes of Health, USA (Grant HL 17754), Japan Tabacco and Salt Public Corporation, and Japan Heart Foundation. 